\title{
Following multi-dimensional type la supernova explosion models to homologous expansion
}

\author{
F. K. Röpke \\ Max-Planck-Institut für Astrophysik, Karl-Schwarzschild-Str. 1, 85741 Garching, Germany \\ e-mail: fritz@mpa-garching.mpg.de \\ Received 21 July 2004 / Accepted 16 November 2004

\begin{abstract}
The last years have witnessed a rapid development of three-dimensional models of type Ia supernova explosions. The next step is to evaluate these models varying the initial parameters and to compare them with observations. To calculate synthetic lightcurves and spectra from numerical models, it is mandatory to follow the evolution up to homologous expansion. We report on methods to achieve this in our current implementation of multi-dimensional type Ia supernova explosion models. The novel scheme is thoroughly tested in two dimensions and a simple example of a three-dimensional simulation is presented. We discuss to what degree the assumption of homologous expansion is justified in these models.
\end{abstract}

Key words. stars: supernovae: general - hydrodynamics - methods: numerical

\section{Introduction}

Type Ia supernova (SN Ia) explosions are commonly attributed to thermonuclear explosions of white dwarf (WD) stars (Hoyle $\&$ Fowler 1960) in a binary system. The precise scenario of these events is, however, controversial (for a recent review see Hillebrandt \& Niemeyer 2000) and it seems possible that different mechanisms contribute to the SN Ia class. The final decision must be made by comparing theoretical models with detailed observations. Substantial progress has been achieved on both sides during the past years. The rapid development of three-dimensional models of thermonuclear supernova explosions (e.g. Hillebrandt et al. 2000; Reinecke et al. 2002a,b,c; Gamezo et al. 2003; Calder et al. 2004) leads naturally to the question of whether they are capable of reproducing observed features of SNe Ia. Systematic parameter studies on the basis of three-dimensional SN Ia explosion models have become possible (Röpke \& Hillebrandt 2004).

For the models by Reinecke et al. (2002a,b,c) synthetic light curves have been calculated with very encouraging results (Sorokina \& Blinnikov 2003). However, for a thorough comparison of SN Ia models with observations via synthetic light curves and spectra, one must follow the explosion simulations up to homologous expansion. The models by Reinecke et al. (2002a,b,c) reach only $t=1.5 \mathrm{~s}$ and also Gamezo et al. (2003) stayed below $t=2 \mathrm{~s}$, although it seems likely that the former are more advanced in the explosion stage, since there the initial flame shape is superposed with stronger spatial perturbations which enhance the development of Rayleigh-Taylor-like instabilities and turbulence.

A further motivation for evolving the explosion models to later times is to explore the effects of slower turbulent combustion regimes (the so-called distributed burning). These apply to low fuel densities reached after $t \sim 1.5 \mathrm{~s}$ by expansion of the WD. The present work focuses on reaching the stage of homologous expansion and the issue of late burning will be addressed in a separate study.

Parametrized one-dimensional SN Ia models (e.g. Nomoto et al. 1984; Thielemann et al. 1986; Höflich et al. 1998) especially with a Lagrangian description of the hydrodynamics - can easily be evolved to homologous expansion. Here, a good approximation is usually assumed after about $10 \mathrm{~s}$. The task is, however, numerically much more challenging for multidimensional models of thermonuclear supernovae.

In the following we report on modifications of the code of Reinecke et al. $(1999,2002 a)$ that enable us to follow the explosion model for much longer times than previous multidimensional simulations. These novel methods are tested in two-dimensional models. For these as well as for an example in three dimensions the approach to homologous expansion is studied. All these models are very simple and known not to produce realistic SN Ia explosions. Therefore they are not suited to judging the underlying astrophysical scenario. Such decisions have to be made based on more advanced models and derived synthetic light curves and spectra. Our simulations are based on the single degenerate Chandrasekhar mass deflagration scenario (see Hillebrandt \& Niemeyer 2000). However, the methods developed here can easily be applied to other models, featuring for instance a delayed detonation.

\section{Homologous expansion in SN la explosions}

A few seconds after ignition of the flame SN Ia explosions are expected to approach self-similar (homologous) expansion, 
which is characterized by a fluid velocity proportional to the radius,

$v(r) \propto r$.

This implies that the relative positions of the fluid elements no longer change. Consider the equation of motion in the Lagrangian formulation,

$\rho \frac{\mathrm{d} v}{\mathrm{~d} t}=-\nabla P-\rho \nabla \Phi$,

with $P, \rho$, and $\Phi$ denoting the pressure, the density and the gravitational potential, respectively. From this equation it follows that no relative change of the velocity of the fluid elements co-moving with the expansion is equivalent to a vanishing pressure gradient and evanescent gravitational force. Hydrodynamical interaction ceases if $\nabla P=0$ and free expansion is reached for $\nabla \Phi \rightarrow 0$.

Another criterion for the approach to homologous expansion can be derived from the energy balance of the explosion process. All energy liberated in the explosion is generated by thermonuclear reactions. While only a tiny fraction of this energy is radiated away giving rise to the observable luminous event, most of it is used to overcome the gravitational binding of the WD star and to accelerate the ejecta. Due to expansion the ejecta are diluted and cooled. Thus, approaching homologous expansion, the gravitational energy and the internal energy should become small compared to the kinetic energy.

\section{Numerical model and modifications}

\subsection{Explosion model}

Our numerical model is based on the scheme proposed by Reinecke et al. (1999) and includes the improvements described by Reinecke et al. (2002a).

The hydrodynamics are modeled by the Euler equations with species conversion and appropriate source terms to take into account nuclear reactions. The numerical solution of these equations is based on the PROMETHEUs implementation (Fryxell et al. 1989) of the Piecewise Parabolic Method (PPM) by Colella \& Woodward (1984).

The equation of state (EoS) models the degenerate matter of the WD star taking into account an electron gas that is degenerate and relativistic to a variable degree, the completely ionized nuclei following the Maxwell-Boltzmann distribution, a photon gas and electron-positron pair creation.

The appropriate description of the nuclear reactions would be given by a full reaction network. Since this is too costly to run concurrently with the explosion model, a simplified description is chosen. Following the approach suggested by Reinecke et al. (2002a) five species are included, namely $\alpha$-particles, ${ }^{12} \mathrm{C},{ }^{16} \mathrm{O},{ }^{24} \mathrm{Mg}$ as a representative of intermediate mass elements, and ${ }^{56} \mathrm{Ni}$ as a representative of iron group nuclei. The composition of the WD prior to the explosion is assumed to be a mixture of ${ }^{12} \mathrm{C}$ and ${ }^{16} \mathrm{O}$. At the initially high densities, burning proceeds to nuclear statistical equilibrium (NSE) composed of $\alpha$-particles and nickel. Depending on temperature and density in the ashes, the fraction of $\alpha$-particles and nickel changes. Once the fuel density drops below $5.25 \times 10^{7} \mathrm{~g} \mathrm{~cm}^{-3}$ due to the expansion of the WD, burning is assumed to terminate at intermediate mass elements. Below $1 \times 10^{7} \mathrm{~g} \mathrm{~cm}^{-3}$ burning enters the regime of distributed turbulent combustion, eventually becoming very slow, and is no longer followed. This may not be a satisfactory approach when the evolution is simulated to later times since it is possible that even the much slower flame contributes to the production of intermediate mass elements. However, this will not affect the production of iron group elements and may therefore be neglected in order to obtain a first-order estimate of the produced nickel mass and a lower limit of the total energy. But for synthetic spectra it may have a significant impact. We will address this issue in a forthcoming study and ignore it in the following, focusing on the hydrodynamical evolution of the models rather than on a detailed nucleosynthetic description.

Since the range of relevant length scales in a SN Ia explosion (from the radius of the WD of $\sim 1000 \mathrm{~km}$ to the width of the flame which is less than a centimeter) is by far too large to be resolved in numerial simulations, the flame evolution has to be modeled by an appropriate effective scheme. This is accomplished by treating the flame as a discontinuity between fuel and ashes. We will call this model representation of the flame "flame front" in the following.

In the so-called flamelet regime of turbulent combustion, which applies to large parts of thermonuclear SN explosions (Niemeyer \& Woosley 1997), the flame is wrinkled by interaction with turbulent velocity fluctuations. These stem from a turbulent cascade, which is generated by large scale buoyancy (Rayleigh-Taylor) instabilities. The interaction with turbulence increases the surface of the flame front and accelerates its propagation. Turbulence dominates flame propagation down to the Gibson scale, which is far below the numerical resolution. Below the Gibson scale, flame propagation proceeds stably in the cellular burning regime and therefore these scales have little effect on the macroscopic flame evolution (Röpke et al. 2003; Röpke et al. 2004a,b).

According to these considerations the discontinuity description not only averages the finite internal flame structure but additionally defines a mean flame location neglecting the wrinkling of the flame at scales below the resolution of the computational grid. In this way it represents a "flame brush" rather than a smooth flame. To determine the turbulent burning velocity of the flame front the sub-grid scale model described by Niemeyer \& Hillebrandt (1995) is applied.

The propagation of the flame front is implemented following the the level set technique by Osher \& Sethian (1988). This technique was extended to deflagration flames by Smiljanovski et al. (1997) and a scheme (the so-called "passive implementation") applicable to SN Ia explosion models was developed by Reinecke et al. (1999). The basic idea is to associate the flame front $\Gamma$ with the zero level set of a signed distance function $G$

$\Gamma:=\{\boldsymbol{r} \mid G(\boldsymbol{r})=0\}, \quad|\nabla G| \equiv 1$.

Its temporal evolution is prescribed by the so-called $G$-equation $\frac{\partial G}{\partial t}=-\boldsymbol{D}_{\mathrm{f}} \cdot \nabla G$ 
The velocity of the front motion is given by

$\boldsymbol{D}_{\mathrm{f}}=\boldsymbol{v}+s_{\mathrm{t}} \boldsymbol{n}$

for the passive implementation with $\boldsymbol{v}$ and $\boldsymbol{n}$ denoting the fluid velocity and normal vector to the flame front, respectively. The turbulent burning velocity $s_{\mathrm{t}}=\sqrt{2 q_{\mathrm{sgs}}}$ is determined by the subgrid-scale energy $q_{\text {sgs }}$ provided by the sub-grid scale turbulence model. With this approach the flame front is described as a sharp discontinuity and topological changes are handled without problems (Reinecke et al. 1999).

\subsection{Co-expanding computational grid}

To evolve numerical models to homologous expansion it is necessary to capture significantly growing length scales in the computational domain. One approach could be to start with a wide-spaced static computational grid and use adaptive mesh refinement to resolve the relevant parts of the domain. This approach will, however, become increasingly expensive with evolution time, since the developing turbulence will fill large parts of the domain in which the grid has to be refined. In explicit schemes the maximum time evolution for each hydro step is given by the CFL criterion (Courant et al. 1928). It states that to ensure stability of the hydrodynamics solver the time step needs to be chosen smaller than the time the fastest possible wave would need to cross a computational grid cell. Thus refining the grid drastically increases the number of hydro steps necessary to evolve the model. The level set method together with the sub-grid scale model provide a high accuracy even at relatively coarse computational grids. Therefore we do not apply adaptive mesh refinement.

The simulations by Reinecke et al. (2002a,b,c) were performed on a grid that was equally spaced in the center of the WD. To capture at least part of the expansion of the WD, the width of the computational cells was exponentially increased in the outer regions resulting in a highly non-uniform grid. In the following we will refer to this implementation as the static grid approach. An advantage of this approach is that the resolution of the flame front is relatively fine as long as it stays in the uniform inner part of the grid. If this is true for the stage of the highest energy generation rate, numerical convergence of the models can be reached already for a grid with 256 cells per spatial dimension (Reinecke et al. 2002c). The static grid approach, however, has a number of disadvantages:

- With the static grid it is very expensive to follow the explosion model to homologous expansion.

- The non-uniformity of the grid is an obstacle to the implementation of the level set method. The necessary reinitialization of the signed distance function $G$ (Sussman et al. 1994) is difficult in the elongated grid cells in the outer regions.

- Some more advanced sub-grid scale models tested in simulations similar to that presented below (Schmidt et al., in preparation) require a uniform computational grid.

- In the enlarged outer computational cells the flame front representation is very coarse. This problem is less severe in models that are confined to only one spatial octant (which was the case in all previous simulations), because the buoyancy-induced burning bubbles developed preferentially along the axes. This is not true if this artificial symmetry constraint is abolished and simulations of the full WD star are performed (Röpke \& Hillebrandt 2005).

Therefore we implemented a computational grid that coexpands with the explosion. Here the grid spacing is allowed to vary and the resulting discretization of the equations of hydrodynamics is neither Eulerian nor Lagrangian. With this scheme it is possible to track the expansion of the WD during the SN Ia explosion with a uniform computational grid. This approach will be denoted as co-expanding grid in the following. It overcomes the drawbacks of the static grid approach.

However, due to the uniformity and the expansion of the grid, the flame front will be less resolved at the stage of maximum energy generation if one starts out with the same grid spacing as in the inner parts of the static grid approach. Therefore it has to be tested which resolution is necessary in the new implementation to reach numerical convergence.

Our approach is based on the "moving grid" technique ${ }^{1}$ by Winkler et al. (1984). This technique was already implemented in the original PROMETHEUS code (Fryxell et al. 1989). Previously, it was mostly used in connection with radiationhydrodynamics codes (e.g. Liebendörfer et al. 2004, 2002; Buchler et al. 1997; Mair et al. 1992; Dorfi \& Feuchtinger 1991). We will give a brief outline of the derivation of the underlying equations following Müller (1994) and Winkler et al. (1984). Let $(\partial / \partial t)$ denote the Eulerian derivative taken with respect to fixed coordinates in the laboratory frame and $(\mathrm{D} / \mathrm{D} t)$ the Lagrangian derivative taken with respect to a definite fluid element. The derivative with respect to fixed values of the moving grid ("moving grid derivative") will be designated $(\mathrm{d} / \mathrm{d} t)$. Analogous to the fluid velocity

$\boldsymbol{v}=\frac{\mathrm{D}}{\mathrm{D} t} \boldsymbol{r}_{\mathrm{e}}$

where $\boldsymbol{r}_{\mathrm{e}}=\boldsymbol{r}_{\mathrm{e}}\left(\boldsymbol{r}_{0}, t\right)$ specifies the position of a definite fluid element, we define the grid velocity

$\boldsymbol{v}_{\text {grid }}=\frac{\mathrm{d}}{\mathrm{d} t} \boldsymbol{r}_{\text {grid }}$

Here, $\boldsymbol{r}_{\text {grid }}$ is the position of a definite set of grid coordinates. The relative velocity between fluid and moving grid is then given by

$\boldsymbol{v}_{\text {rel }}=\boldsymbol{v}-\boldsymbol{v}_{\text {grid }}$

Lagrangian and Eulerian coordinates are special cases of the moving grid for $\boldsymbol{v}_{\text {grid }}=\boldsymbol{v}$ and $\boldsymbol{v}_{\text {grid }}=0$, respectively. The Eulerian and Lagrangian time derivatives of the density $a$ of an extensive quantity are related by

$\frac{\mathrm{D}}{\mathrm{D} t} a=\frac{\partial}{\partial t} a+v \boldsymbol{\nabla} a$

${ }^{1}$ Originally, Winkler et al. (1984) called this technique adaptive mesh. To avoid confusion with adaptive mesh refinement methods, we will denote it moving grid technique, following Müller (1994). 
with $\boldsymbol{\nabla}$ taken with respect to Eulerian coordinates. Similarly, $\frac{\mathrm{d}}{\mathrm{d} t} a=\frac{\partial}{\partial t} a+\boldsymbol{v}_{\text {grid }} \boldsymbol{\nabla} a$

relates the moving grid derivative to the Eulerian derivative.

Introducing the Jacobian $J_{\mathrm{f}}$ of the transformation between coordinates defining the initial volume of a fluid element $\mathrm{d} V_{\text {fluid }}^{0}$ and the volume $\mathrm{d} V_{\text {fluid }}=J_{\mathrm{f}} \mathrm{d} V_{\text {fluid }}^{0}$ of the same fluid element at later times it is straightforward to derive the Euler expansion formula (e.g. Müller 1994)

$\frac{\mathrm{D} \ln J_{\mathrm{f}}}{\mathrm{D} t}=\boldsymbol{\nabla} \cdot \boldsymbol{v}$

which leads to the Reynolds transport theorem

$\frac{\mathrm{D}}{\mathrm{D} t} \int_{V_{\text {fluid }}}\left(a \mathrm{~d} V_{\text {fluid }}\right)=\int_{V_{\text {fluid }}}\left\{\frac{\partial a}{\partial t}+\boldsymbol{\nabla} \cdot(\boldsymbol{v} a)\right\} \mathrm{d} V_{\text {fluid }}$

Analogously one obtains the moving grid expansion formula

$\frac{\mathrm{d} \ln J}{\mathrm{~d} t}=\boldsymbol{\nabla} \cdot \boldsymbol{v}_{\text {grid }}$

where $J$ now denotes the Jacobian of the transformation between a moving grid volume $\mathrm{d} V_{\text {grid }}=J_{\mathrm{f}} \mathrm{d} V_{\text {grid }}^{0}$ and its initial volume $\mathrm{d} V_{\text {grid }}^{0}$.

This leads to the moving grid transport theorem (see e.g. Müller 1994)

$\frac{\mathrm{d}}{\mathrm{d} t} \int_{V_{\text {grid }}}\left(a \mathrm{~d} V_{\text {grid }}\right)=\int_{V_{\text {grid }}}\left\{\frac{\partial a}{\partial t}+\boldsymbol{\nabla} \cdot(a v)\right\} \mathrm{d} V_{\text {grid }}$.

Inserting the general form of a balance equation in Eulerian form

$\frac{\partial}{\partial t} a=-\nabla \cdot(a v)+s(a)$

where $s(a)$ denotes the source term, into Eq. (4) one obtains

$$
\begin{aligned}
\int_{V_{\text {grid }}} s(a) \mathrm{d} V_{\text {grid }}= & \frac{\mathrm{d}}{\mathrm{d} t} \int_{V_{\text {grid }}} a \mathrm{~d} V_{\text {grid }} \\
& +\int_{V_{\text {grid }}} \boldsymbol{\nabla} \cdot\left[a\left(\boldsymbol{v}-\boldsymbol{v}_{\text {grid }}\right)\right] \mathrm{d} V_{\text {grid }} \\
= & \frac{\mathrm{d}}{\mathrm{d} t} \int_{V_{\text {grid }}} a \mathrm{~d} V_{\text {grid }}+\iint_{\partial V_{\text {grid }}} a \boldsymbol{v}_{\text {rel }} \cdot \mathrm{d} \boldsymbol{S} .
\end{aligned}
$$

With the appropriate quantities $a$ and source terms $s(a)$ this defines the set of equations of hydrodynamics that is solved numerically.

\section{Simulation setup}

The two-dimensional simulations were carried out on one quadrant assuming axial symmetry and mirror symmetry to the other half space. In the three-dimensional simulations, only one spatial octant was calculated assuming mirror symmetry to the other octants. All simulations with a co-expanding grid started with a computational domain of $\left[2.02 \times 10^{8} \mathrm{~cm}\right]^{2}$ discretized on the respective number of computational cells. Reflecting boundary conditions were applied on all sides of the domain.
The explosion simulation was started with a WD near the Chandrasekhar mass limit in hydrostatic equilibrium. Its initial temperature was set to $T_{0}=5 \times 10^{5} \mathrm{~K}$. For the composition a $50 \%$ mixture of ${ }^{12} \mathrm{C}$ and ${ }^{16} \mathrm{O}$ was chosen. The central density $\rho_{\mathrm{c}}$ was set to $2.9 \times 10^{9} \mathrm{~g} \mathrm{~cm}^{-3}$. With these initial values a WD model was constructed by integrating the equations of hydrostatic equilibrium using the EoS described above (see Reinecke 2001). An important point is that due to the limited range of the EoS this integration was stopped as soon as a density of $10^{-3} \mathrm{~g} \mathrm{~cm}^{-3}$ was reached. This value was kept constant in the outer regions ("pseudo-vacuum"), which thus cannot maintain hydrostatic equilibrium. The resulting fluid motions in these parts of the computational domain leave the physical evolution of the explosion unaffected, since here the masses are very small. As will be discussed below, they can, however, lead to numerical difficulties. A WD of the mass of $2.797 \times 10^{33} \mathrm{~g}$ $\left(1.406 M_{\odot}\right)$ resulted from the setup procedure.

The flame was ignited near the center of the WD in a sphere which was superimposed by toroidal perturbations to accelerate the growth of Rayleigh-Taylor like instabilities. In this way, the initial flame structure extended to a radius of $\sim 180 \mathrm{~km}$. It corresponds to the $c 3$ model of Reinecke et al. (2002c). This initial condition is rather artificial. However, the exact way in which the flame formation proceeds is not yet precisely known (Garcia-Senz \& Woosley 1995; and Woosley et al. 2004, provide approaches), and it seems likely that it cannot be resolved in the kind of simulations presented in the following. Therefore one has to find an averaged initial flame prescription. For the exemplary cases, our simple model will suffice, but the initial condition of the burning front remains an open question of SN Ia explosion models.

An issue that deserves some consideration is the prescription of the grid expansion velocity. The goal is to follow the evolution of the WD and thus the radial velocity of the edge of the star has to be tracked. However, in our setup as described above, it is problematic to define the edge of the WD. Neither the pressure nor the temperature vanish here. A certain low density is also not a good indicator of the WD's edge since the overall density drops with expansion and especially the low density of the ashes can become comparable to that "outside" of the star. We therefore followed the $1.4 M_{\odot}$ mass shell. This was achieved by defining a belt that includes $0.006 M_{\odot}$ around this mass shell and determining the mean fluid velocity inside this belt. According to this mean velocity the grid coordinates were expanded self-similarly so that the $1.4 M_{\odot}$ mass shell was located at a fixed position at our computational grid. By tracking a very high mass shell one can prevent the star from reaching the domain boundaries even in case of strong asphericities.

\section{Two-dimensional simulations}

\subsection{Comparison with static grid calculations}

In Fig. 1 the flame front morphologies in a co-expanding grid calculation are compared to a static grid simulation at different epochs of the explosion process. Both simulations start out from the same flame shape and were carried out on an [256] ${ }^{2}$ cell domain. Until $t=0.3 \mathrm{~s}$ the evolution of both flame fronts 

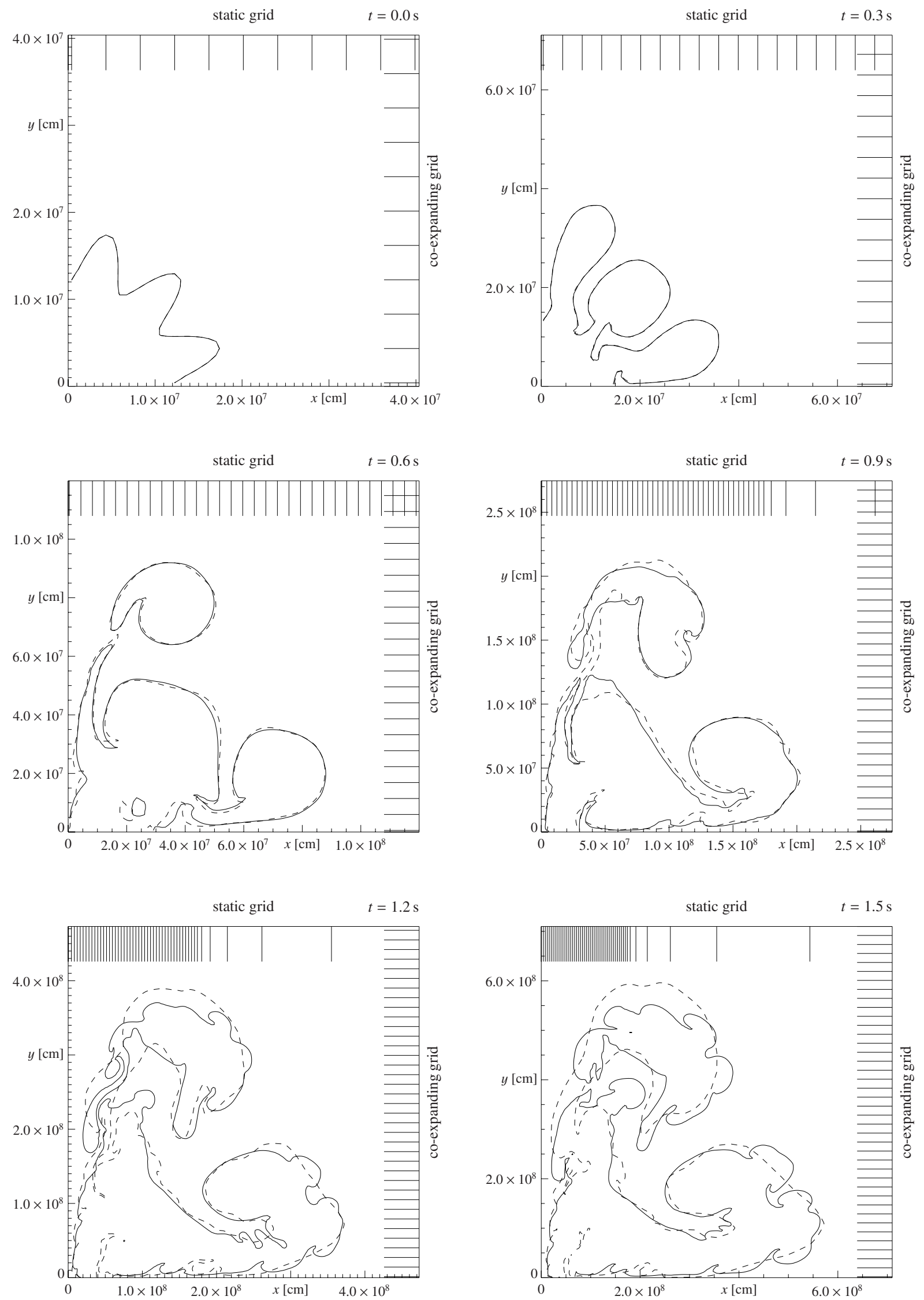

Fig. 1. Comparison of the flame front evolution in a simulation with co-expanding computational grid (solid) with a static grid simulation (dashed). The right and the upper axis ticks indicate the location of every fifth grid cell for the co-expanding and the static grid, respectively. 


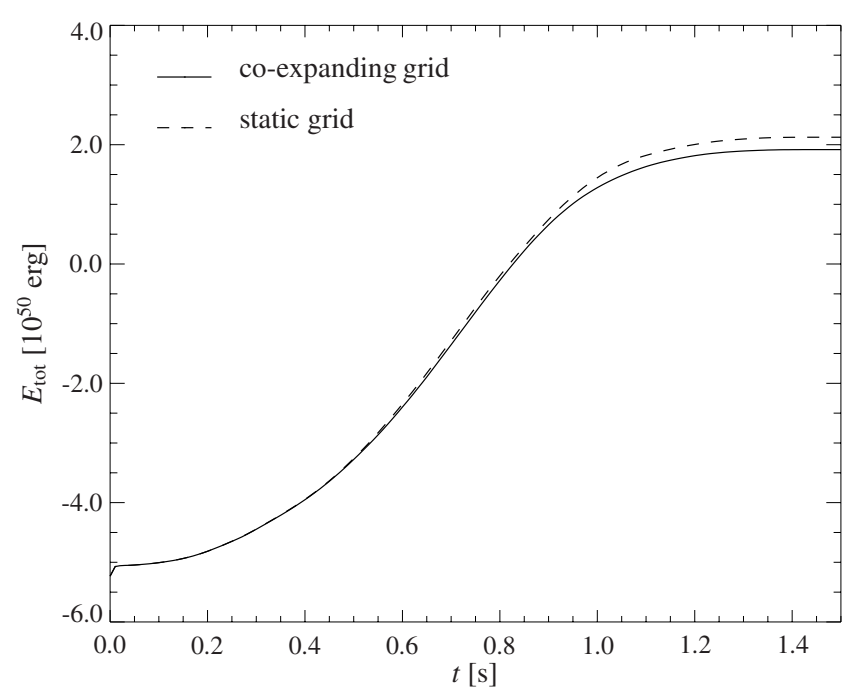

Fig. 2. Total energy in the simulations with co-expanding and static grid.

is almost identical. This is trivially the case because the grid spacing is the same in the part of the domain that is occupied by the flame front. In the co-expanding grid simulation the grid spacing is changed according to the velocities around the mass shell of $1.4 M_{\odot}$. This shell is reached by the first shock wave due to the prompt incineration of the material behind the initial flame after $t \approx 0.3 \mathrm{~s}$. From that moment on the grid keeps expanding self-similarly. Therefore its spacing at $t=0.6 \mathrm{~s}$ is larger than that of the inner part of the static grid. The better resolution of the flame front in the static grid case leads to a slight deviation of the flame front shape evolution (cf. Fig. 1). However, at $t=0.9 \mathrm{~s}$ the flame front in the static grid simulation has already entered the part of the domain with exponentially increasing grid spacing and consequently its outer parts are less resolved than the flame front in the co-expanding grid simulation. Therefore the flame front in the latter case develops more features in the outer parts (see the plots corresponding to $t=1.2 \mathrm{~s}$ and $t=1.5 \mathrm{~s}$ in Fig. 1).

Summarizing the results plotted in Fig. 1, we find that the flame front in the co-expanding grid simulation develops less structure in the inner parts and more features in the outer parts than the corresponding flame front in the static grid simulation. However, the overall flame front evolution is very similar.

The total energies produced by the two models are plotted in Fig. 2. Both models produced too little energy to account for a SN Ia explosion. This is expected for two-dimensional simulations with the chosen flame setup. The co-expanding grid simulation produced slightly less energy than the static grid case. This effect can again be attributed to the different evolution of the resolution of the flame front. Figure 3 shows clearly that the energy production rate reaches a maximum at around $0.7 \mathrm{~s}$ (the initial high value of the energy generation originates from the instantaneous incineration of the material behind the initial flame). At this time, however, the grid spacing at the flame front location in the co-expanding case is already wider than the one in the static grid approach.

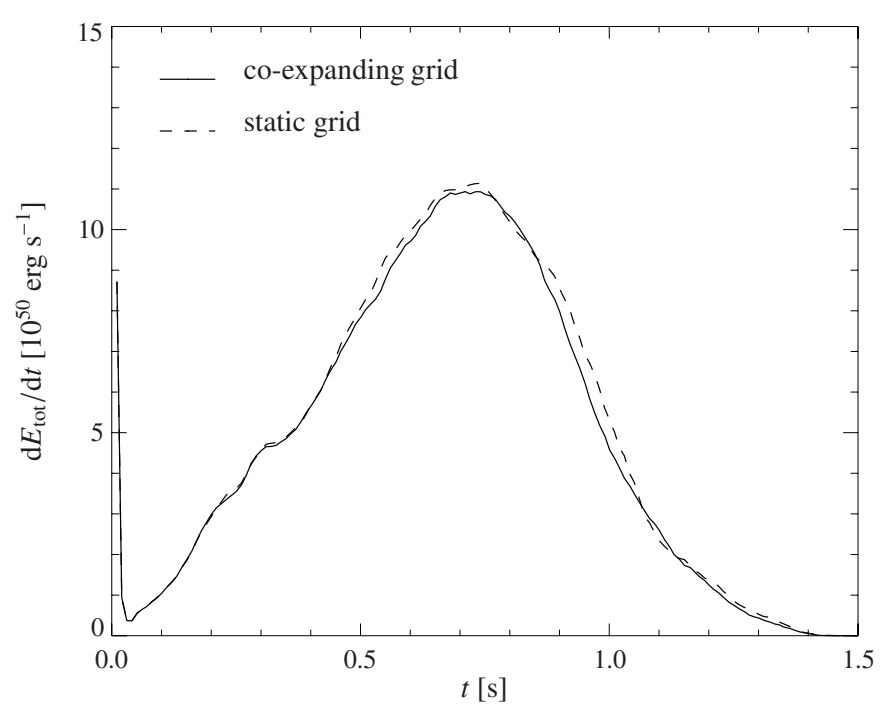

Fig. 3. Energy production rate in the simulations with co-expanding and static grid.

From our comparison we conclude that the co-expanding grid scheme provides a reliable and robust model of the SN Ia deflagration phase. Small differences compared to the previously used static grid approach can be attributed to the different resolutions of the flame front at different epochs of the explosion process.

\subsection{Numerical convergence}

Numerical convergence is a basis to judge credibility of simulations. In the scenario under consideration, the flame front is unstable and affected by turbulence on scales that reach far below the resolution that is achievable in multi-dimensional simulations. From this point of view, numerical convergence in the details of the flame morphology cannot be reached. However, in our models convergence of global quantities (like the total energy generation or the production of burning products) is expected to result from the interplay of large-scale flame front features with the sub-grid scale model. Ideally, a lack of resolution of large-scale features in the flame front representation should be compensated for by an increased turbulent flame propagation velocity determined by the sub-grid scale approach. Of course, a certain threshold of resolution will need to be exceeded to reach this regime in our numerical implementation.

Our final goal is to simulate $\mathrm{SNe}$ Ia in three spatial dimensions. However, currently it is computationally too expensive to perform three-dimensional convergence tests. We therefore take the approach of Reinecke et al. (2002c) and perform the resolution study in two dimensions. From this we conclude on the behavior in three dimensions. For our convergence tests we conducted a series of two-dimensional simulations with resolutions of $[64]^{2},[128]^{2},[256]^{2},[512]^{2},[1024]^{2}$, and $[2048]^{2}$ grid cells. The flame fronts and densities after $1.5 \mathrm{~s}$ (when burning has ceased) are visualized in Fig. 4. The model with the lowest resolution (Fig. 4a) shows a peculiar behavior. In the better resolved simulations the large-scale features resemble each 

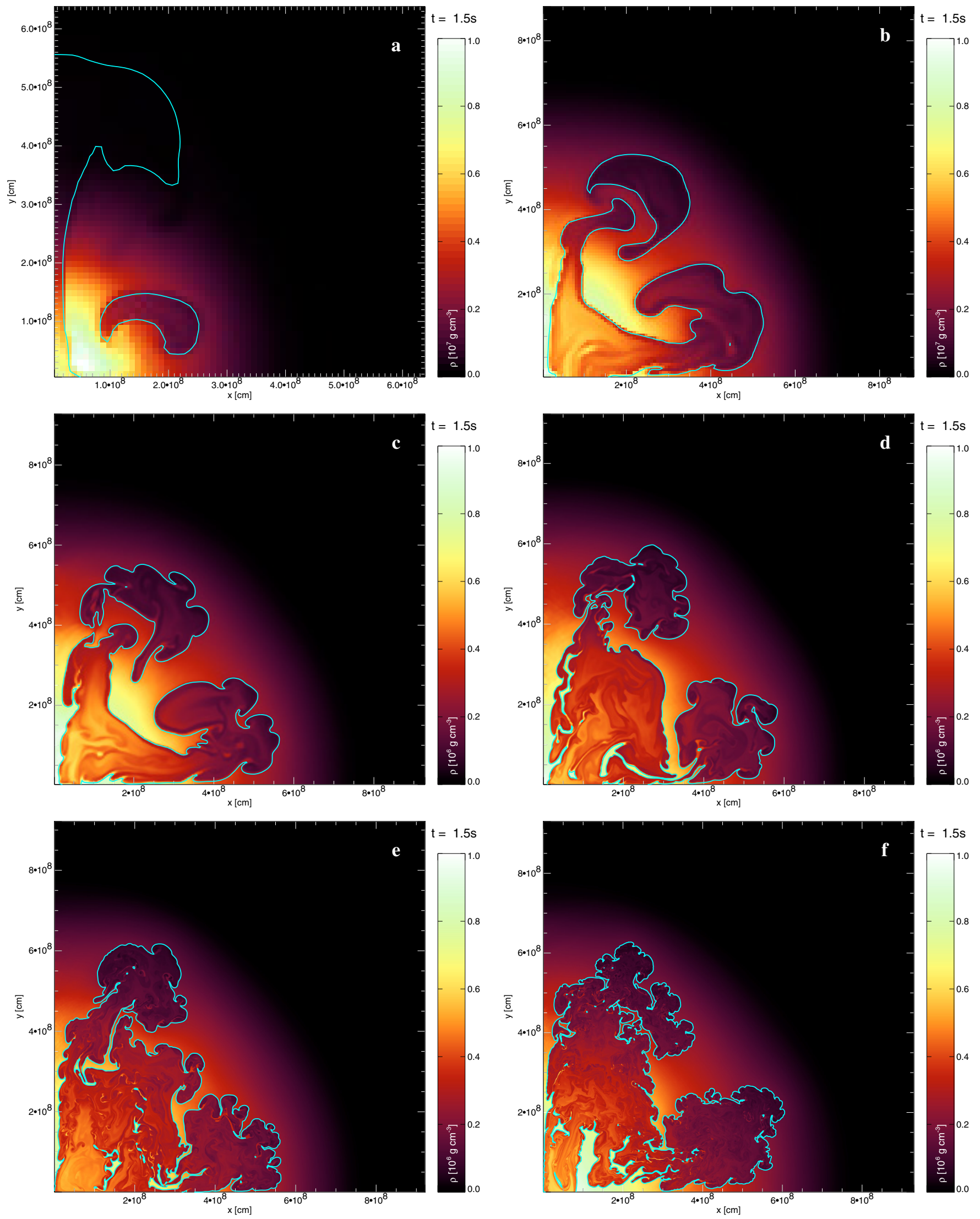

Fig. 4. Two-dimensional SN Ia simulations with different domain discretizations: a) $[64]^{2}$; b) $[128]^{2}$; c) $[256]^{2}$; d) $[512]^{2}$; e) $[1024]^{2}$; and f) $[2048]^{2}$ cells. 


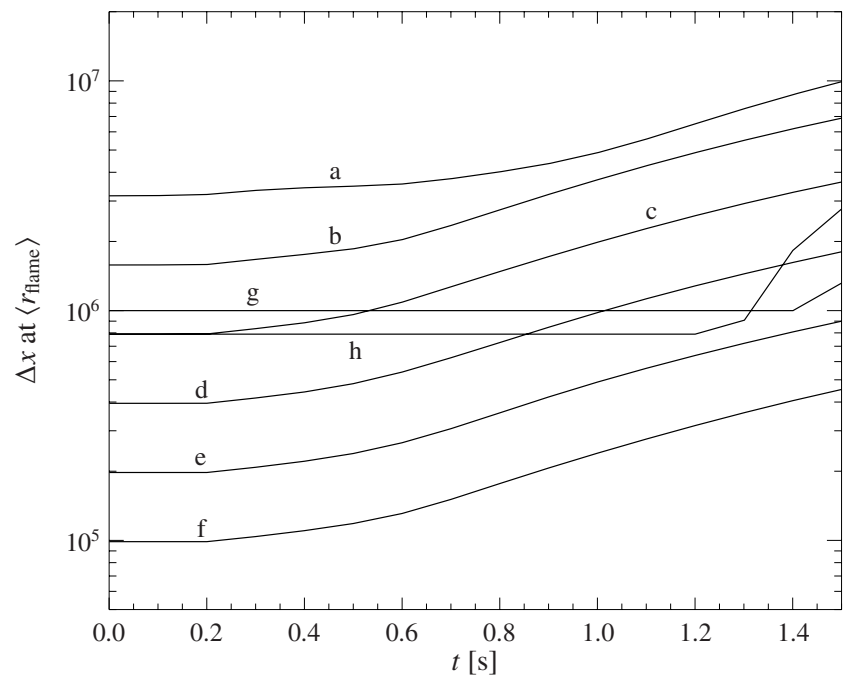

Fig. 5. Resolution of the flame front in co-expanding simulations with (a) $[64]^{2}$ cells; (b) $[128]^{2}$ cells; (c) $[256]^{2}$ cells; (d) $[512]^{2}$ cells; (e) $[1024]^{2}$ cells; (f) $[2048]^{2}$ cells, and in static grid simulations with (g) $[256]^{2}$ cells and an inner grid spacing of $10^{6} \mathrm{~cm}$; (h) $[256]^{2}$ cells and an inner grid spacing of $7.9 \times 10^{5} \mathrm{~cm}$.

other to a certain degree. But also here the details differ. The buoyancy instability of the flame front gives rise to a nonlinear evolution of the large-scale flame features. The particular realization of this is sensitive to small perturbations and thus the different numerical noise in the models may lead to variations in the large-scale features. Thus, even in numerically converged models a small scatter of the global characteristics is possible.

For the static grid simulation Reinecke et al. (2002c) found numerical convergence in the energy production for grid spacings of the inner part of the domain below $\Delta x \approx 10^{6} \mathrm{~cm}$. However, for the late stages, when the flame front enters the nonuniform part of the domain, the energy production failed to converge in that test.

This points to an important issue in our simulations. Neither in the co-expanding nor in the static grid approach the resolution of the flame front is constant. For the latter the flame front resolution is constant only in the early stages of the explosion but decreases rapidly once the flame enters the non-uniform part of the computational grid. In the co-expanding grid approach, the resolution of the flame front decreases steadily. This is demonstrated in Fig. 5, where the resolution of the flame front is plotted against time. In this plot, graph $g$ corresponds to a static grid simulation that has reached numerical convergence according to Reinecke et al. (2002c). It is obvious that only co-expanding grid simulations with $[1024]^{2}$ or more grid cells resolve the flame front better at all times. It needs to be emphasized at this point, that for the co-expanding grid model the situation is further complicated by the fact that the grid resolution is adapted to the expansion of the star. Thus, more vigorous explosions will decrease the numerical resolution faster.

The total energies produced in our simulations with different domain discretizations are plotted in Fig. 6. The runs with $[64]^{2}$ and $\left[128^{2}\right]$ cells show a peculiar behavior. The simulations with a resolution of [512] $]^{2}$ cells and better seem to be numerically converged. It is interesting to note that the

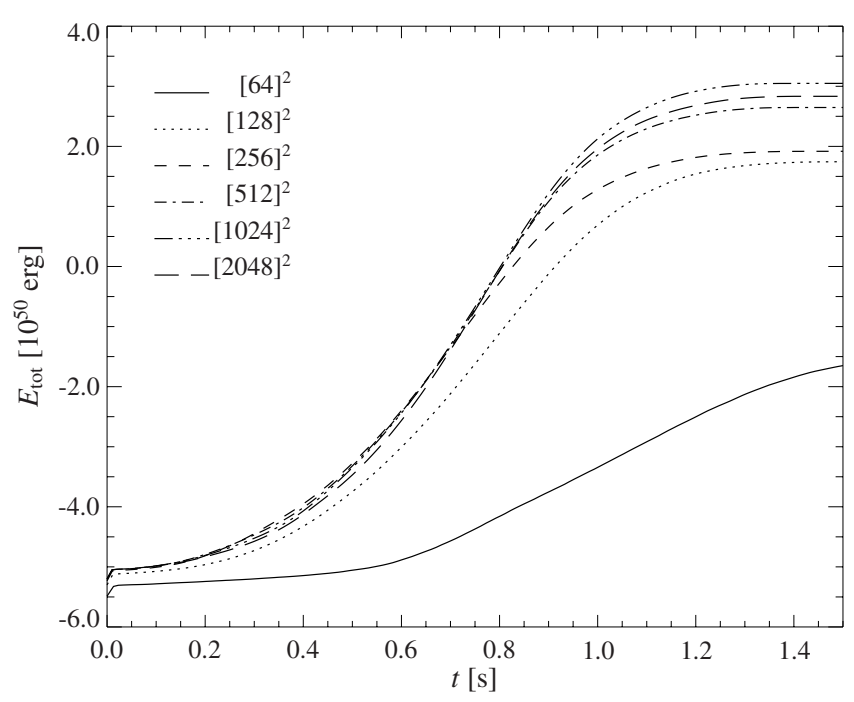

Fig. 6. Total energy in simulations with co-expanding grid for different numbers of computational grid cells.

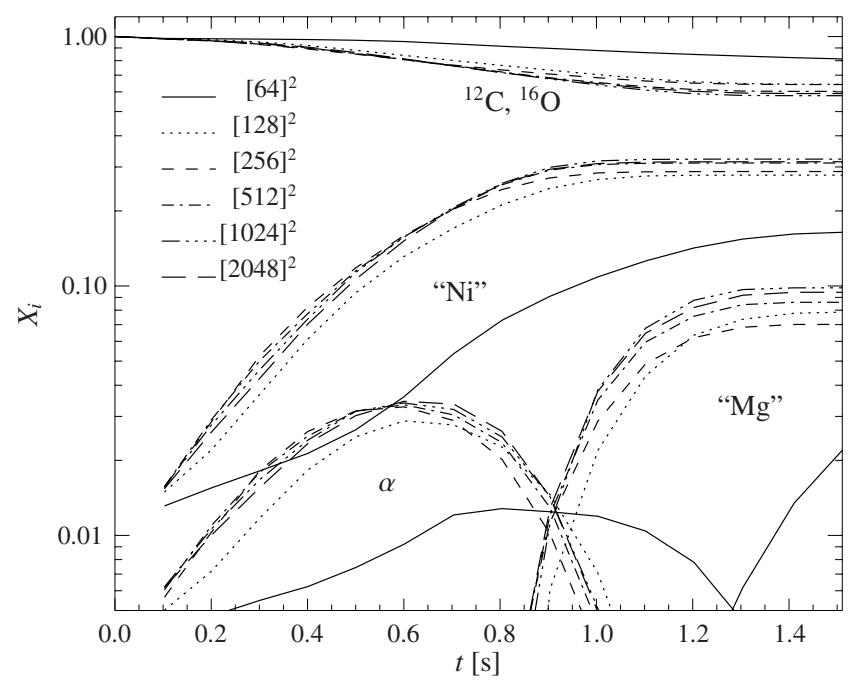

Fig. 7. Evolution of the chemical composition in simulations with coexpanding grid for different numbers of computational grid cells.

model with $[2048]^{2}$ cells releases slightly less energy than the $[1024]^{2}$ cells model. As discussed above, this can be attributed to the slightly different large-scale realization of the flame front which is obvious from a comparison of Figs. $4 \mathrm{e}$ and 4f. The model with a resolution of $[256]^{2}$ cells is converged in the early stages but the energy production falls behind the higher resolved runs for $t \lesssim 0.7 \mathrm{~s}$. This is similar to what was found by Reinecke et al. (2002c) and can be attributed to the decreasing resolution of the flame front.

The evolution of the chemical composition in our simulations is determined by the propagation velocity of the flame front and the fuel density at the flame front and thus closely related to the expansion of the WD, which in turn depends on the flame front propagation. The temporal evolution of the composition is plotted in Fig. 7. It is obvious that in the models with $[64]^{2}$ and $[128]^{2}$ cells the chemical evolution deviates considerably from the better resolved runs. Here an insufficiently resolved flame front burns less material but also leads to a 
slower expansion of the star (cf. Fig. 4). For the other models the production of iron group elements can be regarded as converged but the $[256]^{2}$ cells simulation shows less intermediate mass elements, which are produced in later stages of the explosion.

This makes the simulation with $[256]^{2}$ cells the most important to judge numerical convergence. Both the evolution of the total energy and the chemical composition in that model suggest that the resolution of the flame front becomes too coarse to be numerically resolved at $t \approx 0.7 \mathrm{~s}$. According to Fig. 5 this corresponds to a resolution of $\sim 15 \mathrm{~km}$.

Our study implies that for two-dimensional simulations this is given for models with more than $[512]^{2}$ cells starting from a resolution of better than $3.95 \mathrm{~km}$. A model with [256] $]^{2}$ (with $(\Delta x)_{\text {ini }}=7.9 \mathrm{~km}$ ) cells will give the correct production of iron group elements but slightly underproduce intermediate mass nuclei. Since three-dimensional simulations explode more strongly than their two-dimensional counterparts (cf. Reinecke et al. 2002a), a somewhat better initial resolution is desirable here. It needs to be emphasized that the resolution is not only crucial for numerical convergence, but also for the implementation of the physical model. Reinecke et al. (2002b) find the most energetic explosions for multi-spot ignition scenarios. To accommodate an adequate number of initial flame spots, one needs, however a rather fine computational grid.

\subsection{Reaching homologous expansion}

Figure 8 depicts the temporal evolution of our SN Ia simulation with $[1024]^{2}$ cells up to $t=10.0 \mathrm{~s}$. Note that the color-coded density range varies in the snapshots. The solid contour represents the zero level set of the $G$-function. This is associated with the flame front at early times, but after $t \sim 2 \mathrm{~s}$ the density of the WD has become so low due to expansion that burning ceases in our description. It is, however, interesting to note that the $G=0$ level set is still a good indicator of the interface between unburnt and burnt material at later times (see snapshot at $t=5.0 \mathrm{~s}$ and compare to the snapshot at $t=10.0 \mathrm{~s}$ where the $G=0$ contour was omitted). The reason for this somewhat surprising behavior is that the turbulent energy in our simulation decreases drastically after about $2 \mathrm{~s}$ This is shown in Fig. 9 where the total sub-grid scale turbulent energy is plotted against time. One has to keep in mind, however, that $q_{\mathrm{sgs}}$ increases with the size of the grid cells, which even moderates the decrease of the turbulent energy plotted in Fig. 9. Hence later than $t \approx 2 \mathrm{~s} q_{\mathrm{sgs}}$ becomes so small that the fluid velocity $v$ dominates in Eq. (3) and consequently the $G=0$ isosurface (isoline) is advected passively with the resolved flow.

Figure 9 again demonstrates numerical convergence for our models with more than $[256]^{2}$ cells. Here the criterion is not an exact match of the value of the turbulent sub-grid scale energy but its temporal evolution. This is similar for the highly resolved simulations while the less resolved runs diverge at early times.

The morphology of the density structures does not significantly change after $t \sim 5 \mathrm{~s}$. This indicates that our simulation approaches homologous expansion. How consistent our simulation at late times really is with the assumption of homologous expansion has to be decided on the basis of the velocity profile and the amplitude of the gradients of pressure and the gravitational potential.

This will be analyzed for our simulation with $[1024]^{2}$ cells. Figure 10 shows the deviation of the angular averaged velocities from relation (1) at different times. The proportionality factor of that relation was obtained from a fit to the velocity profile at $t=10 \mathrm{~s}$. The velocity deviations are plotted against the distance from the WD's center. This distance is expressed in widths of computational cells $\Delta x$ to allow comparison; because of the uniform grid the physical distance scales directly with $\Delta x$. We note that the deviations from homologous expansion decrease drastically. Except from the region in the very center the deviation at $t=10 \mathrm{~s}$ stays below $5 \%$. The static grid simulations ended at $t=1.5 \mathrm{~s}$. Here deviations still reach $10 \%$. The maximal values and the mean values of the pressure gradients are given in Table 1. These quantities also decrease drastically with time. From $t=0.6 \mathrm{~s}$ (where the energy generation rate peaks) to $t=1.5 \mathrm{~s}$ they differ less than a magnitude while from $t=1.5 \mathrm{~s}$ to $t=10.0 \mathrm{~s}$ they drop two magnitudes. The evolution of the gradients of the gravitational potential (see Table 1) shows the same trend.

We conclude that our simulations at $t \approx 10 \mathrm{~s}$ provide a fair approximation of homologous expansion. This is supported by Fig. 11 (solid curve) showing that at $t \approx 10 \mathrm{~s}$ the kinetic energy of our model is almost one order of magnitude larger than the sum of the internal and gravitational energies.

Due to the co-expanding grid the computational costs to reach homologous expansion are moderate. The expanding grid spacing leads to an increase in the time steps satisfying the CFL criterion. For the Godunov-scheme used to solve the equations of hydrodynamics in our implementation this criterion states for the time step $\Delta t$

$$
\Delta t \leq \Delta x\left(\max _{i}\left\{\left|\boldsymbol{v}_{i}\right|+c_{\mathrm{s}, i}\right\}\right)^{-1},
$$

where the index $i$ runs over all computational cells and $v$ and $c_{\mathrm{s}}$ denote the fluid velocity and the sound speed, respectively. The CFL criterion is necessary but not sufficient to ensure stability of the scheme. To guarantee stability we reduce the time step by a factor of 0.8 in our implementation.

The evolution of the time steps taken in our simulation with $[256]^{2}$ cells is plotted in Fig. 12. Two points should be noted here. First, the time step is very low between $\sim 0.30 \mathrm{~s}$ and $\sim 0.38 \mathrm{~s}$. The reason for this is that the shock wave generated by the initial flame setup reaches the WD's surface and propagates into the surrounding pseudo-vacuum. Here high velocities are generated so that computational cells outside the star dominate in the CFL criterion. However, once the first shock wave has crossed the WD, it starts to expand and the computational grid co-expands accordingly. With the reflecting outer boundaries this leads to a damping of the motions in the cells filled by the pseudo-vacuum and the time step recovers to larger values. The second point to note here is that the increase of the time step due to the expansion makes it possible to reach from $2 \mathrm{~s}$ to $10 \mathrm{~s}$ with only $\sim 10 \%$ more hydro steps than calculating up to $2 \mathrm{~s}$. 

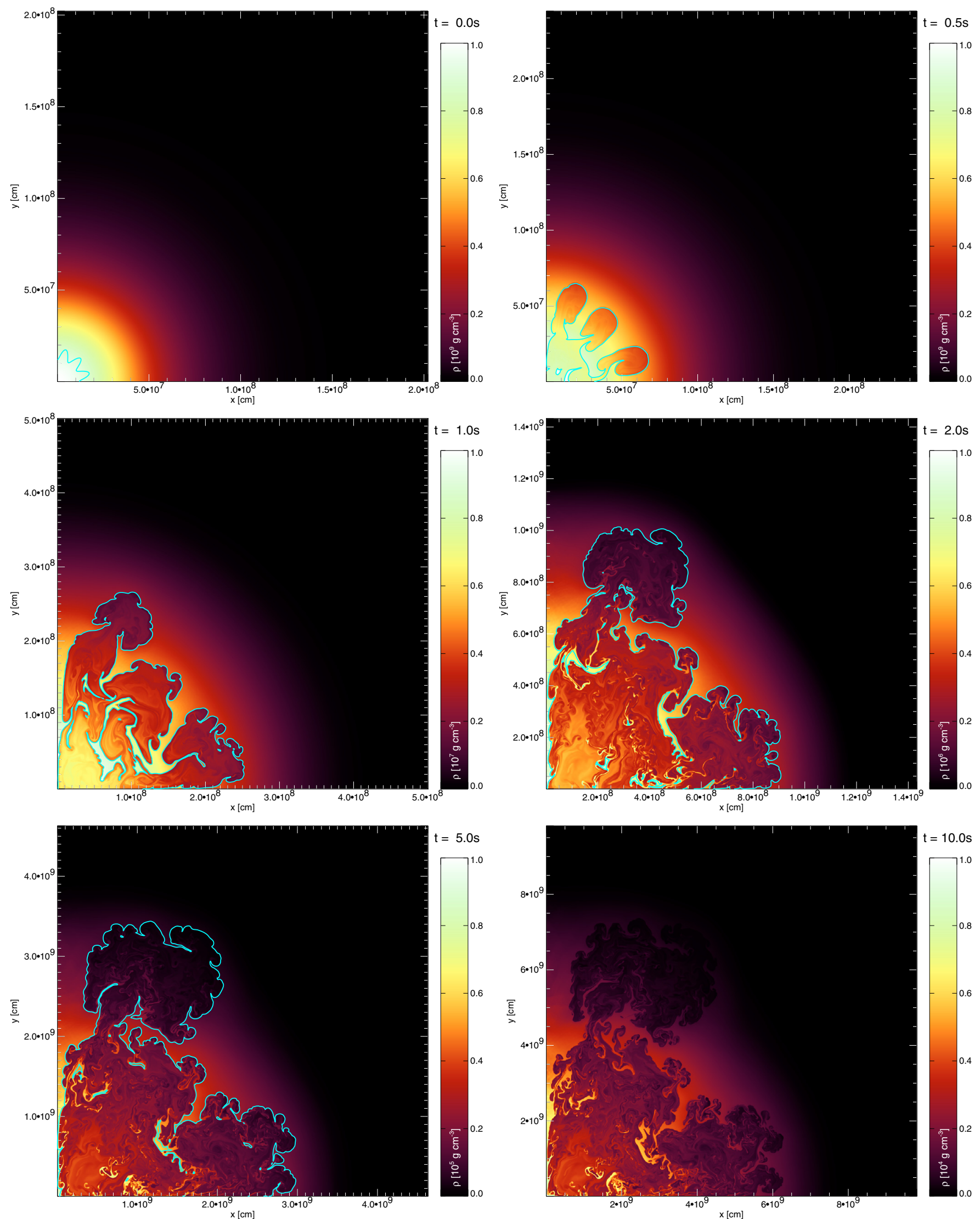

Fig. 8. Two-dimensional SN Ia simulation with $[1024]^{2}$ cells. 


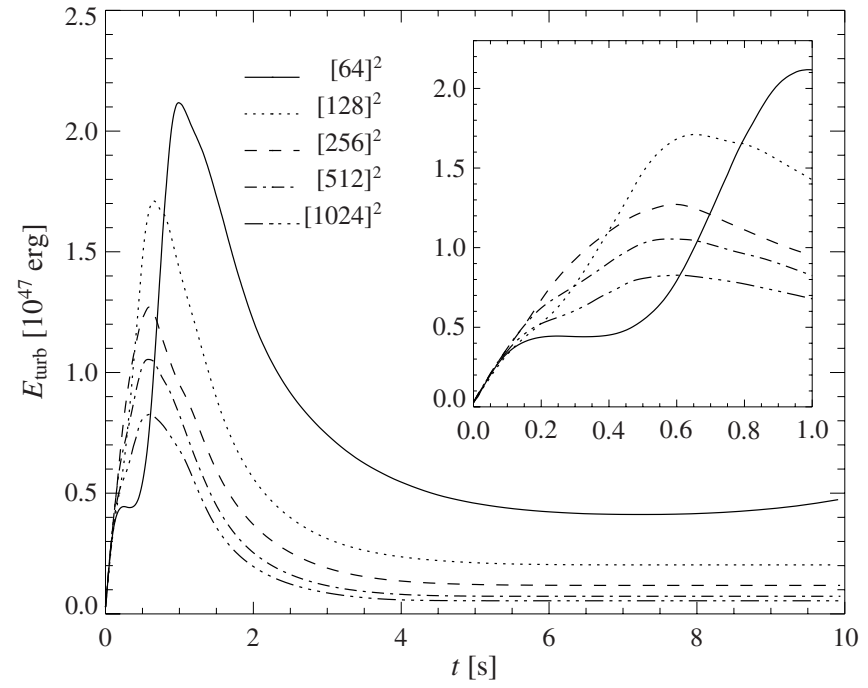

Fig. 9. Evolution of the turbulent energy in simulations with coexpanding grid for different numbers of computational grid cells.

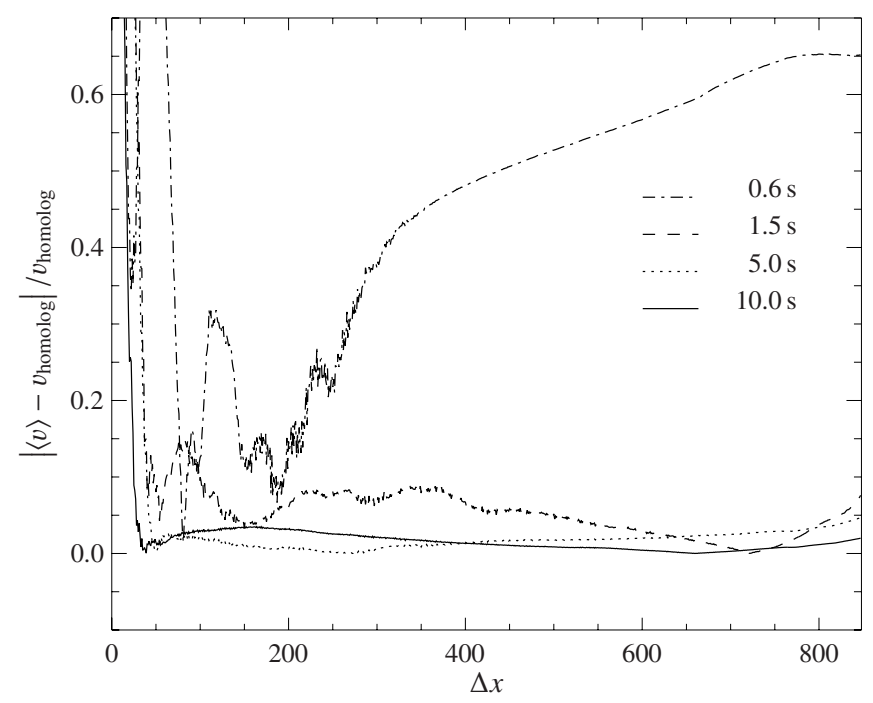

Fig. 10. Deviation of the angular averaged velocity from homologous expansion at different times in the two-dimensional simulation with $[1024]^{2}$ cells.

Table 1. Pressure gradients and gradients of the gravitational potential in the two-dimensional $[1024]^{2}$ cells simulation at different times.

\begin{tabular}{rlll}
\hline \hline$t[\mathrm{~s}]$ & $\begin{array}{l}\max |\boldsymbol{\nabla} p| \\
{\left[\mathrm{dyn} \mathrm{cm}^{-3}\right]}\end{array}$ & $\begin{array}{l}\langle|\nabla p|\rangle \\
{\left[\mathrm{dyn} \mathrm{cm} \mathrm{cm}^{-3}\right]}\end{array}$ & $\begin{array}{l}\max |\boldsymbol{\nabla} \Phi| \\
{\left[\mathrm{cm} \mathrm{s}^{-2}\right]}\end{array}$ \\
\hline 0.6 & $9.44 \times 10^{7}$ & $1.17 \times 10^{7}$ & $8.17 \times 10^{9}$ \\
1.5 & $6.85 \times 10^{7}$ & $2.95 \times 10^{6}$ & $4.05 \times 10^{8}$ \\
5.0 & $3.82 \times 10^{5}$ & $8.74 \times 10^{4}$ & $1.74 \times 10^{7}$ \\
10.0 & $1.27 \times 10^{5}$ & $3.40 \times 10^{4}$ & $4.09 \times 10^{6}$ \\
\hline
\end{tabular}

\section{A three-dimensional simulation}

With the scheme described in the previous sections we performed a three-dimensional test simulation. The computational domain was discretized into [256 $]^{3}$ cells starting with a grid spacing of $7.9 \times 10^{5} \mathrm{~cm}$ and the flame setup corresponded to the three-dimensional version of the $c 3$-model by

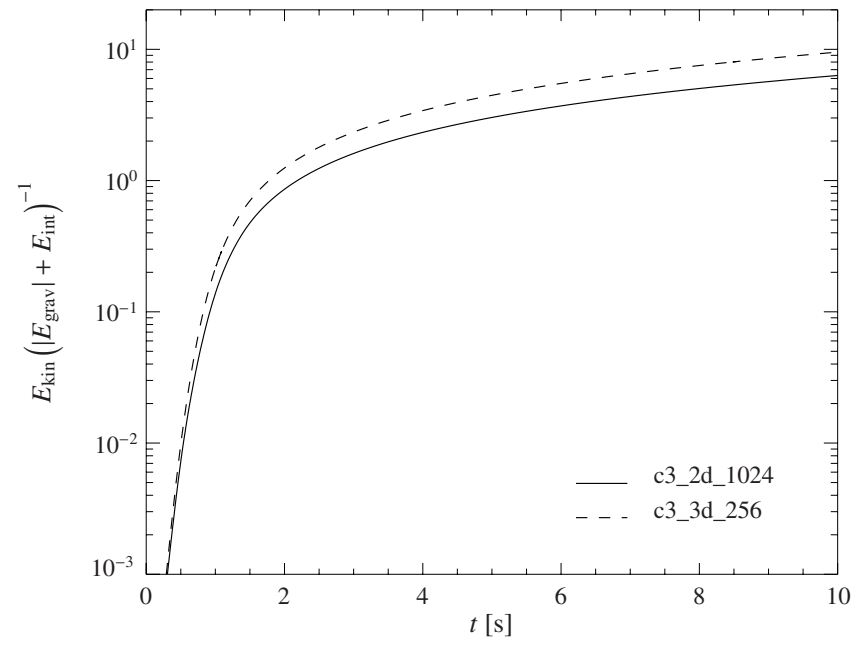

Fig. 11. Temporal evolution of the ratio of the kinetic energy to the sum of internal and gravitational energy for the two-dimensional model with $[1024]^{2}$ cells (solid curve) and the three-dimensional model with $[256]^{2}$ cells (dashed curve).

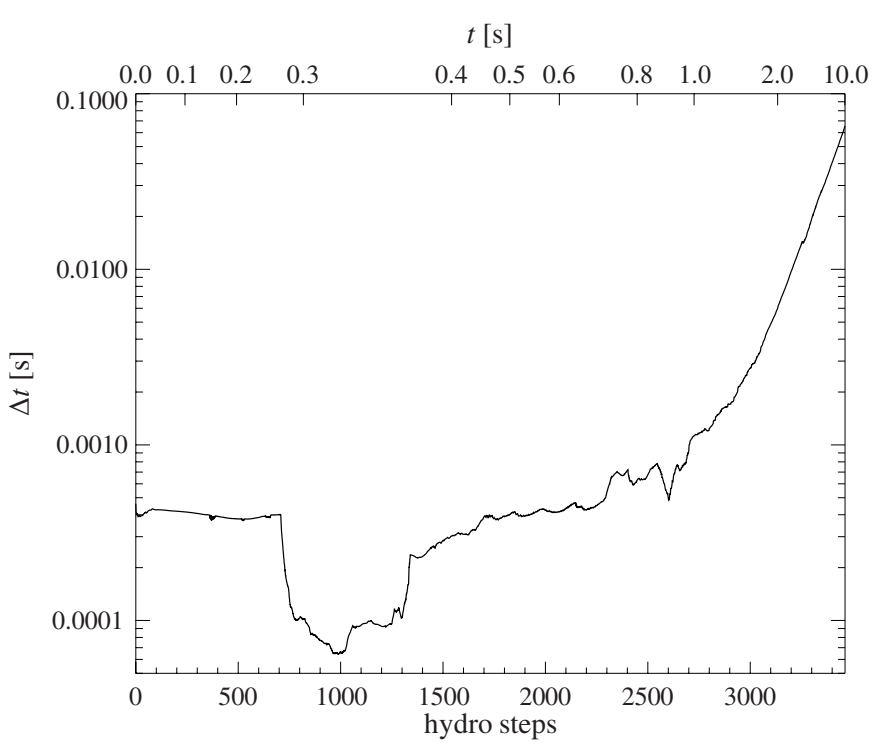

Fig. 12. Time steps in the simulation with $[256]^{2}$ cells.

Reinecke et al. (2002b,c). Snapshots from the simulations are shown in Fig. 13. The rendered isosurface corresponds to the zero level set of $G$. As discussed above it represents the flame front at early stages and still provides an approximate separation between fuel and ashes after $t \sim 2 \mathrm{~s}$.

The early evolution of the flame front is very similar to that found by Reinecke et al. (2002b,c). The initial axial symmetry is broken in the same way. The late stages show more structure due to the better resolution of the co-expanding grid in the outer parts of the domain. Our study in the previous section indicated that the simulation is close to homologous expansion already after $2 \mathrm{~s}$. In the three-dimensional simulation, the morphology of the $G=0$ isosurface still changes. The outer bubbles grow hiding the inner structures. This may have impact on the light curves and the spectra derived from such models. From $t=5 \mathrm{~s}$ to $t=10 \mathrm{~s}$ the visible changes are only marginal. 

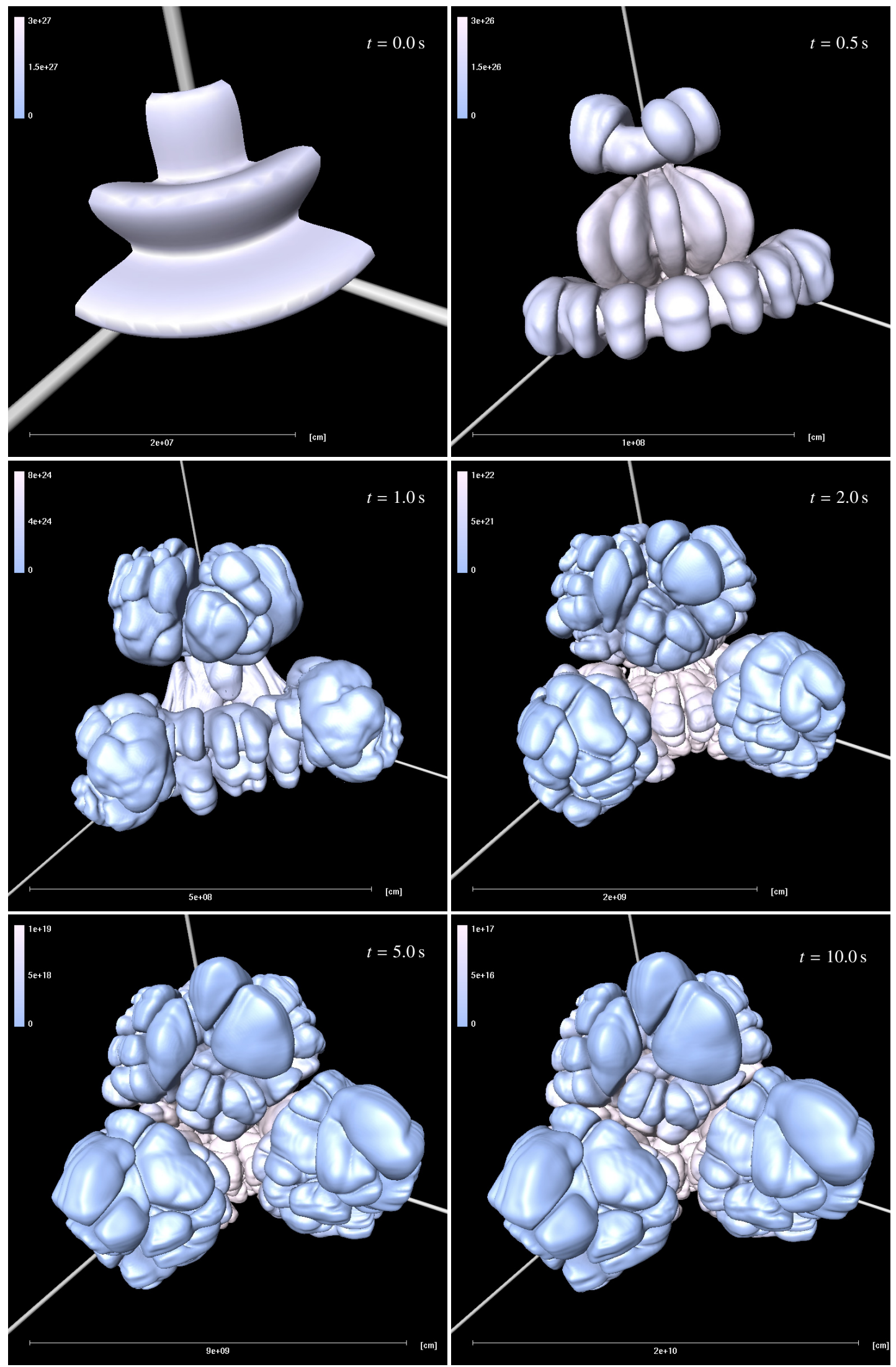

Fig. 13. Three-dimensional SN Ia simulation. The isosurface corresponds to $G=0$ and the color-coding indicates the value of the sub-grid scale energy providing a measure of the propagation velocity of the front. The scale gives an impression of the expansion and corresponds to the plane through the origin of the coordinate system. 


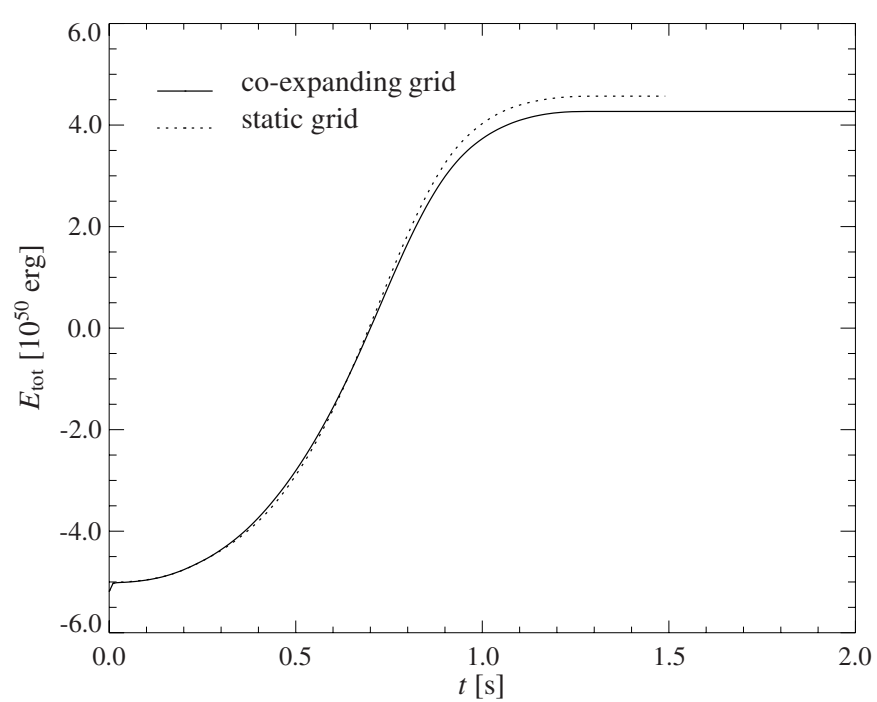

Fig. 14. Comparison of the total energies of our three-dimensional simulation with co-expanding grid and the $c 3 \_3 d \_256$ model (static grid) by Reinecke et al. (2002b,c).

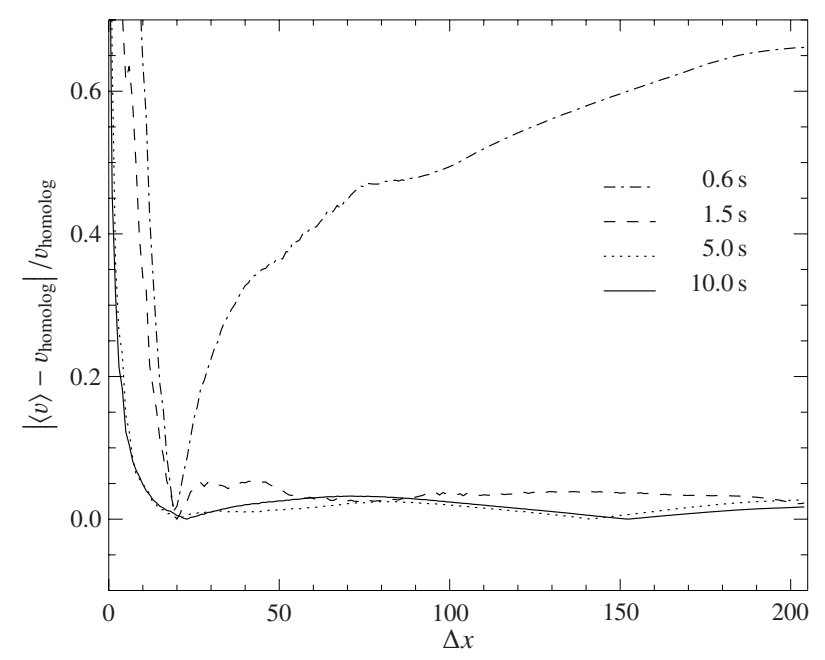

Fig. 15. Deviation of the angular averaged velocity from homologous expansion at different times in the three-dimensional simulation.

The energetics of the model is compared to the c3_3d_256 model $^{2}$ by Reinecke et al. $(2002 \mathrm{~b}, \mathrm{c})$ in Fig. 14. As in the two-dimensional simulations the differences between the static grid and the co-expanding grid simulations can be attributed to the varying resolution of the flame front in the latter.

Figure 15 shows the deviation from a self-similar velocity profile in our three-dimensional simulation. We note that it approaches a relation consistent with Eq. (1) faster than the twodimensional simulations. This can at least partly be attributed to the more energetic explosion resulting in three dimensions, giving rise to higher expansion velocities. Figure 11 corroborates this interpretation showing an accelerated conversion of the energy forms to kinetic energy in the three-dimensional

${ }^{2}$ The data was kindly provided by M. Reinecke. In this model the grid spacing at the inner part of the computational domain was $\Delta x=10^{6} \mathrm{~cm}$.
Table 2. Pressure gradients and gradients of the gravitational potential in the three-dimensional simulation at different times.

\begin{tabular}{rlll}
\hline \hline$t[\mathrm{~s}]$ & $\begin{array}{l}\max |\boldsymbol{\nabla} p| \\
{\left[\mathrm{dyn} \mathrm{cm}^{-3}\right]}\end{array}$ & $\begin{array}{l}\langle|\nabla p|\rangle \\
{\left[\mathrm{dyn} \mathrm{cm}^{-3}\right]}\end{array}$ & $\begin{array}{l}\max |\nabla \Phi| \\
{\left[\mathrm{cm} \mathrm{s}^{-2}\right]}\end{array}$ \\
\hline 0.6 & $5.49 \times 10^{7}$ & $4.02 \times 10^{6}$ & $6.93 \times 10^{9}$ \\
1.5 & $5.57 \times 10^{6}$ & $1.50 \times 10^{6}$ & $3.16 \times 10^{8}$ \\
5.0 & $1.45 \times 10^{5}$ & $4.74 \times 10^{4}$ & $1.49 \times 10^{7}$ \\
10.0 & $5.70 \times 10^{4}$ & $1.90 \times 10^{4}$ & $3.59 \times 10^{6}$ \\
\hline
\end{tabular}

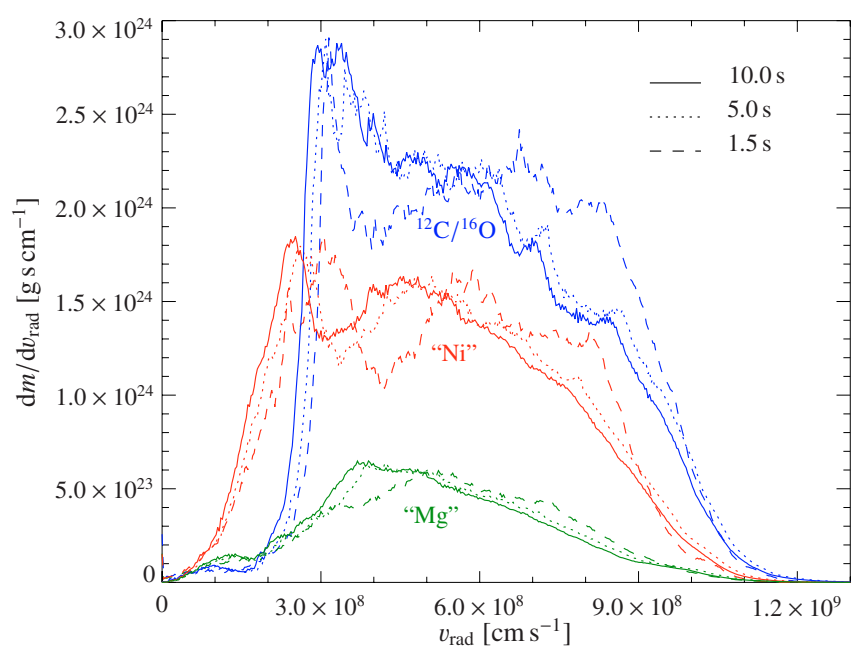

Fig. 16. Distribution of the species in radial velocity space. "Ni" and "Mg" denote the iron group elements and the intermediate mass elements, respectively.

model. The gradients of the pressure and the gravitational potential are given in Table 2 . The maximum value of $|\nabla \Phi|$ develops similar to the two-dimensional case while the mean of the pressure gradient amounts to roughly half the value obtained in the two-dimensional simulation. As in the twodimensional model, the pressure gradient drops significantly between $t=1.5 \mathrm{~s}$ and $t=5.0 \mathrm{~s}$.

The distributions of chemical species in radial velocity space for different times are shown in Fig. 16. The changes between $t=5.0 \mathrm{~s}$ and $t=10.0 \mathrm{~s}$ are small. The profile is somewhat shifted toward smaller velocities which corresponds to a deceleration of the ejecta. This is not surprising since the gravitational potential is small, but not vanishing. In contrast, between $t=1.5 \mathrm{~s}$ and $t=10.0 \mathrm{~s}$ we observe substantial changes in the profiles. All three species tend to pile up at lower velocities, diluting the high-velocity components.

We conclude that our scheme is applicable to threedimensional simulations. The expansion of the computational grid according to the tracked $1.4 M_{\odot}$ mass shell did not cause any difficulties. Homologous expansion in three-dimensional simulations will be reached somewhat quicker than in twodimensional models.

\section{Conclusions}

We analyzed the approach of multi-dimensional SN Ia models to homologous expansion. To this end a novel scheme 
with a co-expanding computational grid was implemented and tested. Taking into account the variable resolution resulting from this scheme, a comparison with previous simulations on a static computational grid showed good agreement of the flame evolution.

A convergence study validated our co-expanding grid approach. Here, the variability of the numerical resolution leads to the conclusion that the grid spacing has to stay below $\sim 15 \mathrm{~km}$ for numerically converged stages of the explosion. For twodimensional simulations one has to start with a domain discretization finer than $[512]^{2}$ cells to resolve the whole explosion process, but a $[256]^{2}$ cell model will already provide the correct production of iron group elements. In the case of threedimensional explosion models a somewhat finer initial resolution is required because the explosions here are more vigorous, resulting in faster expansion.

Applying our co-expanding grid scheme, it was possible to follow the explosion significantly longer than in any previous multi-dimensional SN Ia simulation with only marginal additional computational expense. From the temporal evolution of the velocity profiles, the energy conversion and the gradients of pressure and gravitational potential we conclude that homologous expansion is reached to a high accuracy at $t \sim 10 \mathrm{~s}$. Although the velocity profile is rather quickly in agreement with Eq. (1), the pressure gradient drops significantly at later times. Therefore the flame morphology still changes until $t \sim 5 \mathrm{~s}$. Outer bubbles grow and hide inner parts of the ejecta. Between $t=1.5 \mathrm{~s}$ and $t=10 \mathrm{~s}$ we observe a significant redistribution of chemical species in velocity space $^{3}$. Thus, for the derivation of synthetic light curves and spectra from SN Ia models it is advisable to follow the evolution of the explosion up to $t \sim 10 \mathrm{~s}$. This is in agreement with parametrized one-dimensional models (e.g. Nomoto et al. 1984; Thielemann et al. 1986; Höflich et al. 1998).

The novel numerical scheme presented here offers a variety of improvements of multi-dimensional SN Ia explosion models. A uniform Cartesian grid alleviates some numerical problems (see Sect. 1) and moreover enables the implementation of more sophisticated sub-grid scale models (Schmidt et al., in preparation). Full star SN Ia explosion models, where the artifical symmetry to the spatial octants is abolished, also gain accuracy from such a computational grid (Röpke \& Hillebrandt 2005). However, it is also possible to combine the co-expanding grid approach with the highly non-uniform grid used in previous static-grid simulations. In this way a high resolution of the flame front (especially in the early stages) will be possible with low computational expenses and initial flame conditions can be tested easily. We will address this question in a subsequent study.

An open issue in the current implementation is the modeling of flame propagation in late stages of the explosion. Here

\footnotetext{
${ }^{3}$ It should be emphasized that the $c 3$ model chosen here is very simple and and not expected to realistically reproduce SN Ia explosions. Figure 16 is only intended to illustrate the changes in the approach to homologous expansion. To assess the astrophysical modeling, more advanced models with different initial flame structures and synthetic spectra are needed.
}

the finite width of the flame is no longer negligible since turbulent eddies start to penetrate the preheat zone of the flame. The so-called flamelet assumption of turbulent combustion breaks down and the flame enters the thin reaction zones regime (for a discussion of the turbuelnt burning regimes see e.g. Peters 1999, and for application to SN Ia explosion see Niemeyer \& Kerstein 1997). It is, however, unclear whether burning in this regime contributes significantly to the explosion results. This issue will be addressed in a forthcoming study.

Acknowledgements. The author gratefully acknowledges helpful discussions with W. Hillebrandt, M. Reinecke, K. Kifonidis and W. Schmidt concerning the numerical implementation and astrophysical modeling. L. Scheck helped with the routines to visualize the 2D color snapshots of our simulations. This work was supported in part by the European Research Training Network "The Physics of type Ia Supernova Explosions" under contract HPRN-CT-2002-00303.

\section{References}

Buchler, J. R., Kollath, Z., \& Marom, A. 1997, Ap\&SS, 253, 139

Calder, A. C., Plewa, T., Vladimirova, N., Lamb, D. Q., \& Truran, J. W. 2004, preprint available [arXiv: astro-ph/0405126]

Colella, P., \& Woodward, P. R. 1984, J. Comp. Phys., 54, 174

Courant, R., Friedrichs, K. O., \& Lewy, H. 1928, Math. Ann., 100, 32

Dorfi, E. A., \& Feuchtinger, M. U. 1991, A\&A, 249, 417

Fryxell, B. A., Müller, E., \& Arnett, W. D. 1989, Hydrodynamics and nuclear burning, MPA Green Report 449, Max-Planck-Institut für Astrophysik, Garching

Gamezo, V. N., Khokhlov, A. M., Oran, E. S., Chtchelkanova, A. Y., \& Rosenberg, R. O. 2003, Science, 299, 77

Garcia-Senz, D., \& Woosley, S. E. 1995, ApJ, 454, 895

Hillebrandt, W., \& Niemeyer, J. C. 2000, ARA\&A, 38, 191

Hillebrandt, W., Reinecke, M., \& Niemeyer, J. C. 2000, in Proceedings of the XXXVth rencontres de Moriond: Energy densities in the universe, ed. R. Anzari, Y. Giraud-Héraud, \& J. Trân Thanh Vân (Thê Giói Publishers), 187

Höflich, P., Wheeler, J. C., \& Thielemann, F. K. 1998, ApJ, 495, 617

Hoyle, F., \& Fowler, W. A. 1960, ApJ, 132, 565

Liebendörfer, M., Rosswog, S., \& Thielemann, F. 2002, ApJS, 141, 229

Liebendörfer, M., Messer, O. E. B., Mezzacappa, A., et al. 2004, ApJS, 150, 263

Mair, G., Hillebrandt, W., Hoeflich, P., \& Dorfi, A. 1992, A\&A, 266, 266

Müller, E. 1994, in Lecture Notes in Physics, Vol. 433, Galactic Dynamics and N-Body Simulations, ed. G. Contopoulos, N. K. Spyrou, \& L. Vlahos (Berlin Heidelberg: Springer-Verlag), 313

Niemeyer, J. C., \& Hillebrandt, W. 1995, ApJ, 452, 769

Niemeyer, J. C., \& Kerstein, A. R. 1997, New Astron., 2, 239

Niemeyer, J. C., \& Woosley, S. E. 1997, ApJ, 475, 740

Nomoto, K., Thielemann, F.-K., \& Yokoi, K. 1984, ApJ, 286, 644

Osher, S., \& Sethian, J. A. 1988, J. Comp. Phys., 79, 12

Peters, N. 1999, J. Fluid Mech., 384, 107

Reinecke, M. A. 2001, Ph.D. Thesis, Technical University of Munich, available at http://tumb1.biblio.tu-muenchen.de/ publ/diss/allgemein.html

Reinecke, M., Hillebrandt, W., Niemeyer, J. C., Klein, R., \& Gröbl, A. $1999, A \& A, 347,724$ 
Reinecke, M., Hillebrandt, W., \& Niemeyer, J. C. 2002a, A\&A, 386, 936

Reinecke, M., Hillebrandt, W., \& Niemeyer, J. C. 2002b, A\&A, 391, 1167

Reinecke, M., Niemeyer, J. C., \& Hillebrandt, W. 2002c, New Astronomy Review, 46, 481

Röpke, F. K., \& Hillebrandt, W. 2004, A\&A, 420, L1

Röpke, F. K., \& Hillebrandt, W. 2005, A\&A, 431, 635

Röpke, F. K., Hillebrandt, W., \& Niemeyer, J. C. 2004a, A\&A, 420, 411

Röpke, F. K., Hillebrandt, W., \& Niemeyer, J. C. 2004b, A\&A, 421, 783

Röpke, F. K., Niemeyer, J. C., \& Hillebrandt, W. 2003, ApJ, 588, 952
Smiljanovski, V., Moser, V., \& Klein, R. 1997, Combustion Theory and Modelling, 1, 183

Sorokina, E., \& Blinnikov, S. 2003, in From Twilight to Highlight: The Physics of Supernovae, ed. W. Hillebrandt, \& B. Leibundgut, ESO Astrophysics Symposia (Berlin Heidelberg: Springer-Verlag), 268

Sussman, M., Smereka, P., \& Osher, S. 1994, J. Comp. Phys., 114, 146

Thielemann, F.-K., Nomoto, K., \& Yokoi, K. 1986, A\&A, 158, 17

Winkler, K.-H., Norman, M. L., \& Mihalas, D. 1984, J. Quant. Spectrosc. Radiat. Transfer, 31, 473

Woosley, S. E., Wunsch, S., \& Kuhlen, M. 2004, ApJ, 607, 921 